\title{
CROSSED POLARISING FILTERS TO MEASURE RELATIVE AFFERENT PUPILLARY DEFECTS: REPRODUCIBILITY, CORRELATION WITH NEUTRAL DENSITY FILTERS AND USE IN CENTRAL RETINAL VEIN OCCLUSION
}

\author{
ANDREW RAMSAY, TOM H. WILLIAMSON, STUART PARKS and DAVID KEATING \\ Glasgow
}

\begin{abstract}
SUMMARY
Measurement of a relative afferent pupillary defect (RAPD) can be carried out by attentuation of light received by the normal eye during the swinging flashlight test. Such measurements may be useful in the management of central retinal vein occlusion (CRVO). In this study a method employing cross polarised filters (CPFs) was tested for reproducibility. In addition the pattern of attenuation of light was compared with that by neutral density filters (NDFs). Finally, the method was used to investigate 74 patients with CRVO. The method was reproducible $(8.7 \%$ variation) and showed exponential attenuation of light (in contrast to linear attenuation by the NDFs). In unilateral CRVO a sensitivity of $71 \%$ and specificity of $69 \%$ for the risk of iris neovascularisation was determined by the presence of $\mathrm{RAPD}$ requiring $20^{\circ}$ of rotation of the CPFs. It is concluded that CPFs provide a useful alternative to NDFs for the measurement of RAPD and have some advantages. They can be employed in the clinical management of patients with CRVO.
\end{abstract}

The identification of a relative afferent pupillary defect (RAPD) is part of the routine ophthalmological examination. Although the swinging flashlight test allows the detection of the presence or absence of such a defect it does not give a measure of the defect's severity. Thompson et al. ${ }^{1}$ have described a method of quantification which involves increasing the attentuation of light entering the unaffected eye by using neutral density filters until no afferent defect is detectable. The density of filter necessary to achieve this is a measure of the severity of the

Correspondence to: Andrew Ramsay, Tennent Institute of Ophthalmology, Church Street, Glasgow G11 6NT,UK.
RAPD. A disadvantage of the method was that a stepwise measurement was required, with steps only as large as the difference between the filter densities available to the examiner. In contrast crossed polarising filters provide a means of continuously varying the light transmitted to the eye by altering the rotation of one axis of polarisation against another. These have also been used to measure RAPD. ${ }^{2}$ Although the inter-observer variability has been tested, the technique's intra-observer reproducibility remained undetermined. In addition the different patterns of attentuation of light by crossed polarising and neutral density filters required illustration. An initial intention of the study was to address these two issues.

Measurement of RAPD with neutral density filters has been employed in the investigation of a number of ocular diseases..$^{3-7}$ In central retinal vein occlusion (CRVO) this method has been used to estimate the risk of iris neovascularisation. ${ }^{8-11}$ In this study crossed polarising filters were applied to the assessment of the condition and a predictive value determined for the development of iris neovascularisation.

\section{RAPD MEASUREMENTS}

The RAPD was measured with a device which consists of two pairs of polarising filters mounted side by side (Stereo Optical, North Kanton Avenue, Chicago, IL; Fig. 1). One of each pair of filters can be rotated in front of the other thereby changing the polarising axis from $0^{\circ}$ to $180^{\circ}$ and attenuating the light transmitted. The device was held in one hand and the patient instructed to gaze at a distant object over the top of the crossed polarising filters, thereby allowing visualisation of the pupils by the observer. 


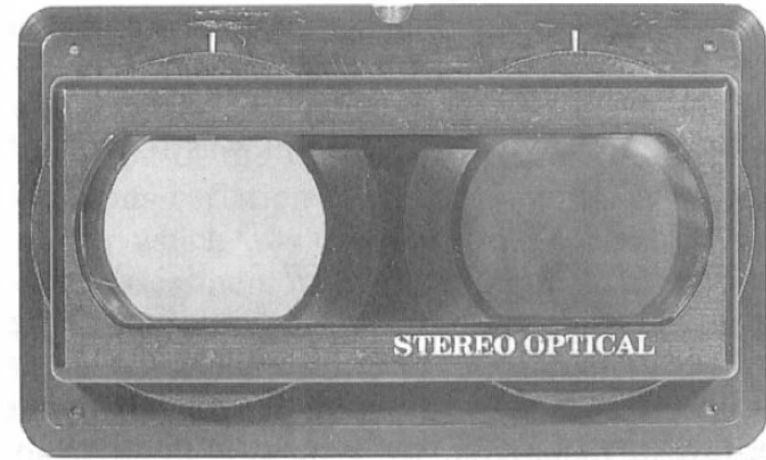

Fig. 1. The crossed polarising filters used (Stereo Optical).

The swinging flashlight test was then performed in a room with dimmed background illumination with the patient fixating on a distant object. ${ }^{1,12}$ The light from an indirect ophthalmoscope set at 6 volts and held 30 $\mathrm{cm}$ from the eye was shone consecutively at each pupil with an angle of $15^{\circ}$ to the visual axis. ${ }^{13}$ The pupillary reaction was observed for 2-3 seconds to allow observation of pupillary behaviour before the rapid transferral of the beam to the other eye. Both crossed polarising filters were initially set at $0^{\circ}$ and the filter rotated on the side opposite to the defect. The end-point for the RAPD measurement was obtained by underestimating and then overestimating the degree of angle rotation required, gradually reducing the interval until the RAPD was neutralised. This end-point occurred when the pupils' initial constriction and subsequent dilatation were asymmetrical. The observer was masked from seeing the polarising angles on the device by covering the calibration marks with tape.

In order to examine the intra-observer reproducibility of the test, 10 patients were examined on two occasions with an interval of 5 minutes between examinations to minimise the effects of retinal bleaching. The mean of the differences between the two examinations was calculated and the standard deviation of the mean difference was used as a measure of the intra-observer variation. ${ }^{14}$ The coefficient of variation of repeated measures (CVRM) was calculated from:

$$
\mathrm{CVRM}=\frac{\mathrm{SD} \text { of mean differences }}{0.5 \times(\text { mean1 } 1-\text { mean } 2)} \times 100 \%
$$

Where mean 1 is the mean of results of the first obervation and mean 2 is the mean of results of the second observation.

\section{CALIBRATION WITH NEUTRAL DENSITY FILTERS}

The relationship of light transmitted through neutral density filters was compared with that passing through the crossed polarising filters at different angles of rotation. A non-coherent light source connected to a stabilised power supply of 9 volts was employed. Combinations of four neutral density filters were placed in front of the light source in order to provide a range of densities in 14 steps of $0.5 \mathrm{log}$ units from 0.5 to $6.5 \log$ units. A radiometer (International Lightmeter 1700 and SED 038 response detector) which measures approximately the same wavelengths of light as the human eye was then placed $30 \mathrm{~cm}$ from the light source. The light transmitted was recorded on four occasions for each combination of neutral density filter to provide values for the mean light transmission. These filters were then substituted for crossed polarising filters and light transmission recorded in a similar fashion at intervals of $5^{\circ}$ of rotation of their axes from $0^{\circ}$ to $90^{\circ}$.

Theoretical values for the transmission of light through both neutral density filters and crossed polarising filters were calculated. The optical density of a neutral density filter was defined as the logarithm of the ratio of incident light to transmitted light and was given by the equation:

$$
D=\log _{10}\left(I D_{0} / I_{\mathrm{t}}\right)
$$

where $D$ is optical density, $I D_{0}$ is incident light and $I_{\mathrm{t}}$ is transmitted light.

The light intensity following transmission through crossed polarising filters conforms to the principle of Malus' law:

$$
I_{\mathrm{t}}=I \Theta_{0} \cdot \cos ^{2}(\Theta) \Theta
$$

where $I_{\mathrm{t}}$ is maximal light intensity and $\Theta$ is the polarising angle.

These two equations were combined, allowing the intensity of light transmitted by the neutral density filter density to be related to that passing through the crossed polarising filters:

$$
D=\log _{10}\left(1 / k \cdot \cos ^{2} \Theta\right)
$$

where $k$ is a constant.

The theoretical and actual values obtained for transmitted light were compared by the least squares fit method.

\section{PREDICTION OF IRIS NEOVASCULARISATION IN CENTRAL RETINAL VEIN OCCLUSION}

RAPD with crossed polarising filters was measured in 74 consecutive patients with CRVO at presentation. Twenty patients had to be excluded from the study for the following reasons: 6 had rubeosis iridis at presentation; 3 had intraocular pressure greater than $30 \mathrm{mmHg}$ in the affected eye; 5 had bilateral vein occlusion; 6 had other ophthalmic disease in the fellow eye ( 2 had retinal detachment, 2 had primary open angle glaucoma, 2 had previous retinal arterial occlusion). Fifty-four patients with a mean age of 64.7 years (range 27-87 years, SD 14.4) remained and consisted of 26 men and 28 women. The mean duration from onset of the occlusion in 
these patients was 3.8 months (range 1 week to 12 months).

Forty-six patients were re-examined 1 year following onset of the occlusion by one of us (T.H.W.); the RAPD was recorded and the presence of iris neovascularisation noted. Two patients who had received prophylactic panretinal photocoagulation were excluded, 1 died and 5 patients were lost to follow-up.

The sensitivity and specificity of the test for the detection of those patients who subsequently developed rubeosis were calculated. The standard deviation of the mean difference between the RAPDs at presentation and at 1 year was calculated to determine whether any change had occurred with time. The difference in Snellen visual acuity at presentation and at 1 year was recorded and a correlation between this and the change in RAPD was examined by Spearman rank correlation.

\section{RESULTS}

Reproducibility

The mean RAPD of the 10 patients was $71^{\circ}(\mathrm{SD}=$

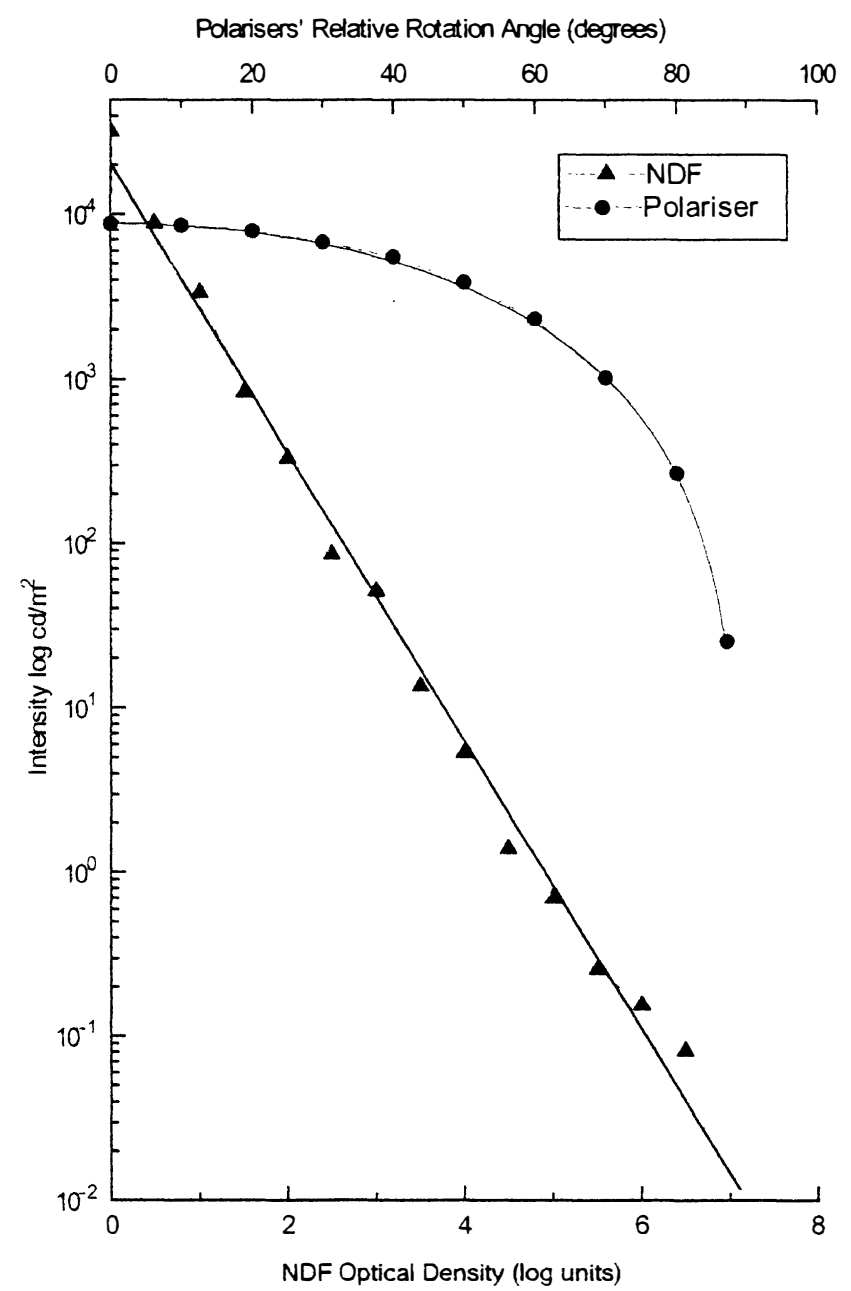

Fig. 2. The intensity of light transmitted by crossed polarising filters (circles) in comparison with neutral density filters (NDF; triangles). $\left.11.5^{\circ}\right)$. There was no significant difference between the first and second readings (two-tailed Student's $t$ test), the mean difference being $0.3^{\circ}$. The coefficient of variation of repeated measures was found to be $8.5 \%$.

\section{Neutral Density Filter Calibration}

The relationship between the attenuation of light from the crossed polarising filters and the neutral density filters is shown in Fig. 2. The attentuation (log intensity of light) by the neutral density filters was related in a linear fashion to the density of the filter. In contrast the angle of rotation of the crossed polarising filters was exponentially related.

The error of the measured attenuation of light to the theoretical attenuation for the neutral density filters was $15 \%$ compared with $1 \%$ for the crossed polarising filters. Using the equations previously described it was possible to determine the value of the constant, $k$. The relationship between the neutral density filters and the crossed polarising filters used in this study was therefore described by:

$$
D=\log _{10}\left[1 / 0.27 \cdot \cos ^{2}(\Theta)\right]
$$

\section{Prediction of Iris Neovascularisation in Central Retinal Vein Occlusion}

Fifty per cent of the patients with CRVO were found to have a RAPD. The mean RAPD measurement in the patients with CRVO at presentation was $24.8^{\circ}$ $(\mathrm{SD}=30.4)$.

The mean RAPD of the 7 patients who developed iris neovascularisation was $33.6^{\circ}(29.8)$. This was significantly greater than the value of $13.4^{\circ}$ (22.4) obtained from the 35 patients who did not develop any neovascular complications (two-tailed Student's $t$-test, $p=0.05)$. Using an RAPD measurement of greater than $20^{\circ}$ as a predictor of the risk of development of iris neovascularisation provided a sensitivity of $71 \%$ and a specificity of $69 \%$. Three patients developed retinal or disc neovascularisation and had RAPDs of $0^{\circ}, 65^{\circ}$ and $70^{\circ}$.

There was no significant difference between the RAPD at first presentation and at 1 year using Student's $t$-test. The standard deviation of the mean differences between the RAPDs at presentation and at 1 year was $32.2^{\circ}$. No correlation was found between the change in RAPD and visual outcome.

\section{DISCUSSION}

The measurement of RAPDs using crossed polarising filters was a reproducible method for quantifying the defect with a small coefficient of variation of repeated measures for intra-observer variation $(8.5 \%)$. Calculation from the results of Rosenberg and Oliva ${ }^{2}$ of inter-observer variation provided a value of $19.7 \%$. It is not surprising that interobserver variation is higher than intra-observer 
variation because the assessment of the null point is subjective and may therefore vary between observers.

Rotation of the crossed polarising filters allowed continuous variation of the attenuation of light - a property which was usefully exploited to provide a precise assessment of RAPD in a clinical setting.

The relationship between the angle of rotation of the filters and the degree of attenuation of light was exponential, observing the principles of Malus' law. Indeed there was an extremely small discrepancy between the measured and calculated values for light attenuation from Malus' law (1\%). In contrast, the neutral density filters provided linear attenuation of light relative to their log density values and were found to have a higher discrepancy $(15 \%)$ from their theoretical model. This may have resulted from the need to combine neutral density filters of different densities, thus introducing a variable number of reflecting and absorbing surfaces.

It should be emphasised that the properties of the attenuation of light by the two types of filters were different. Therefore the numerical values attributed to the RAPD from the two methods, that is density for neutral density filters and rotational angle for crossed polarising filters, are not directly related. Results from the two techniques can be compared only by means of observation of a graph such as Fig. 2 or by consideration of an equation, for example:

$$
D=\log _{10}\left[1 / 0.27 \cdot \cos ^{2}(\Theta)\right]
$$

In this study the calculated constant $(0.27)$ probably accounted for the reflection and absorption of light from the casings of the crossed polarising filters. It should be noted that this will be different for devices other than the ones used.

Crossed polarising filters may be less useful with particularly severe RAPDs requiring high angles of rotation. Observation of Fig. 2 shows that above $80^{\circ}$ small rotations produced particularly large changes in the attenuation of light. In the clinical setting this may not constitute a practical problem; for example in this study only 2 of the 54 patients with CRVO had defects measured at greater than $80^{\circ}$.

Fifty per cent of the patients with CRVO had measurable RAPDs. The patients who subsequently developed iris neovascularisation had more severe RAPDs than those who did not develop this complication. Measurement of RAPD by crossed polarising filters with a cut-off of $20^{\circ}$ provided a specificity of $69 \%$ and a sensitivity of $71 \%$ for the development of iris neovascularisation. This was equivalent to using a $0.62 \mathrm{log}$ units neutral density filter calculated from the equation above. Hayreh $e t$ $a l^{8}$ used neutral density filters and a cut-off of $1.2 \mathrm{log}$ units, which produced a specificity of $70 \%$ and a sensitivity of $89 \%$. The apparent difference in the values for neutral density filters probably reflects a difference in the methodologies in the studies.

Using our methods, crossed polarising filters provided a method of comparable sensitivity and specificity to some of the alternatives such as electroretinography, fluorescein angiography and colour Doppler imaging. ${ }^{10,11,14-24}$ Whereas other methods require sophisticated equipment this technique utilises simple, readily available apparatus without the need for specially trained personnel. It has the added advantages of being inexpensive, quick to perform and non-invasive. Disadvantages include the requirement for a healthy fellow eye and mobile pupils. In this investigation of CRVO the use of the method was precluded for various reasons in 20 of 74 patients. These exclusions are similar to those of Hayreh et al., ${ }^{8}$ who excluded 19 patients with bilateral CRVO and 12 with pupillary abnormalities from a total of 128 patients. Although RAPD measurement could be used in a significant proportion of CRVO patients, other methods may be required in those patients in whom the measurement cannot be made.

Examination of the values for RAPD from followup of the patients showed that the defect varied with time after the onset of CRVO because the 30\% coefficient of repeat measures was greater than can be explained by intra-observer variation of $10 \%$. The change in RAPD, however, could not be related to changes in visual function in these patients.

\section{CONCLUSION}

The measurement of RAPDs by crossed polarising filters is a reproducible method. Crossed polarising filters produce a continuous, exponential attenuation of light in contrast to the linear attenuation by neutral density filters. In unilateral central retinal vein occlusion measurement of the RAPD was of modest predictive value for the risk of iris neovascularisation.

Key words: Central retinal vein, Polarising filter, Pupillary light reflex, Retinal vein occlusion.

\section{REFERENCES}

1. Thompson HS, Corbett JJ, Cox TA. How to measure the RAPD. Surv Ophthalmol 1981;26:39-42.

2. Rosenberg ML, Oliva A. The use of crossed polarising filters in the measurement of the RAPD. Am J Ophthalmol 1990;110:62-5.

3. Bovino JA, Burton TC. Measurement of the RAPD in retinal detachment. Am J Ophthalmol 1980;90:19.

4. Takahashi T, Hohki T, Entani S, Yamashita H, Shiba K. Optic tract syndrome with RAPD. Jpn J Ophthalmol 1991;35:325-30.

5. Lam BL, Thompson S. A unilateral cataract produces a RAPD in the contralateral eye. Ophthalmology 1990;97:334-7.

6. Stanley JA, Baise GR. The swinging flashlight test to detect minimal optic neuropathy. Arch Ophthalmol 1968;80:769-71. 
7. Johnson LN, Hill RA, Bartholomew MJ. Correlation of afferent pupillary defect with visual loss on automated perimetry. Ophthalmology 1988;95:1649-55.

8. Hayreh SS, Klugman MR, Beri M, Kimura AE, Podhajsky P. Differentiation of ischaemic from nonischaemic central retinal vein occlusion during the early acute phase. Graefes Arch Clin Exp Ophthalmol 1990;228:201-17.

9. Grey RH, Bloom PA. Retinal ischaemia and RAPDs in central retinal vein occlusions. Eur $\mathrm{J}$ Ophthalmol 1991;1:85-8.

10. Morrell AJ, Thompson DA, Gibson JM, Kritzinger EE, Drasdo N. Electrophysiology as a prognostic indicator of neovascularisation in CRVO. Eye 1991;5:362-8.

11. Servais GE, Thompson HS, Hayreh SS. RAPD in central retinal occlusion. Ophthalmology 1986;93:3013.

12. Thompson HS. Pupillary signs in the diagnosis of optic nerve disease. Trans Ophthalmol Soc UK 1976;96:377.

13. Browning DJ, Tiedeman JS. The test light affects quantitation of the afferent pupillary defect. Ophthalmology 1987;94:53-5.

14. Bland JM, Altman DG. Statistical methods for assessing agreement between two methods of clinical measurement. Lancet 1986;2:307-10.

15. Baxter GM, Williamson TH. Colour Doppler flow imaging in central retinal vein occlusion: a new diagnostic technique? Radiology 1993;187:847-50.

16. Breton ME, Quinn GE, Keene SS, Dahmen JC, Brucker AJ. Electroretinogram parameters at pre- sentation as predictors of rubeosis in central retinal vein occlusion patients. Ophthalmology 1989;96:134352.

17. Gass JDM. In: Gass JD, editor. Stereoscopic atlas of macular diseases: diagnosis and treatment, 3rd ed. St Louis: CV Mosby, 1987: 422-53.

18. Hayreh SS. So-called 'central retinal vein occlusion'. II. Venous stasis retinopathy. Ophthalmologica 1976;172:14-37.

19. Hayreh SS, Klugman MR, Beri M, Kimura AE, Podhajsky P. Differentiation of ischemic from nonischemic central retinal vein occlusion during the early acute phase. Graefes Arch Clin Exp Ophthalmol 1990;28:201-17.

20. Kaye SB, Harding SP. Early electroretinography in unilateral central retinal vein occlusion as a predictor of rubeosis iridis. Arch Ophthalmol 1988;106:353-6.

21. Laatikainen L, Kohner EM. Fluorescein angiography and its prognostic significance in central retinal vein occlusion. Br J Ophthalmol 1976;60:411-8.

22. Sabates R, Hirose T, McMeel JW. Electroretinography in the prognosis and classification of central retinal vein occlusion. Arch Ophthalmol 1983;101:232-5.

23. Welch JC, Augsburger JJ. Assessment of angiographic retinal capillary nonperfusion in central retinal vein occlusion. Am J Ophthalmol 1987;103:761-6.

24. Williamson TH, Baxter GM. Central retinal vein occlusion, an investigation by colour Doppler imaging: blood velocity characteristics and prediction of iris neovascularisation. Ophthalmology 1994; 101:1362-72. 